Didier Bottineau - CNRS, MoDyCo, Université Paris Ouest - Nanterre - La Défense

\title{
Concordance temporelle et concordance modale dans l'énoncé breton
}

\author{
Gouzout a ouiemp e oac'h ur pezh fall, Aotrou Rastapopoulos. \\ Bremañ e ouzomp ivez ez eus eur lous pezh ac'hanoc'h! \\ « Nous savions que vous étiez une canaille, Monsieur Rastapopoulos. \\ Maintenant, nous savons également que vous êtes un malotru! ${ }^{1}$
}

La concordance des temps a été relativement peu étudiée pour le breton : elle est décrite sommairement par les grammaires pédagogiques (Trepos 2001, Chalm 2008), précisément par les grandes grammaires descriptives (Kervella 1947, Favereau 1997) et quelques rares études (Fave 1998, Heinecke 1999), comme en témoigne l'absence de ce thème dans la bibliographie ARBRES $^{2}$ de M. Jouitteau sur le site du Laboratoire de Linguistique Formelle ; elle n'a pas fait l'objet d'un questionnement théorique consistant. Pourtant, cette question apparaît essentielle pour la compréhension du breton et instructive dans une perspective comparatiste ou typologique pour au moins les raisons suivantes :

- le système verbo-temporel : le breton possède comme le français un présent, un passé simple lennas « lut », un imparfait lenne « lisait», les temps composés (parfait: lennet am eus "j'ai $\mathrm{lu} \gg$ ) et surcomposés correspondants, très utilisés en breton : lennet am eus bet kalzig ' $\mathrm{j}$ 'ai beaucoup eu lu' «j'ai beaucoup lu ») ; un futur, également utilisé comme subjonctif présent lenno «lira $/$ 《lise »; un potentiel lennfe «lise » / «lirait» et un irréel lennje «lise » / « lût »/ « lirait » (temps simples tous les deux, chacun étant combinable avec le parfait). Tous ces temps sont utilisables en indépendante comme en subordonnée, ce qui neutralise la distinction mode / temps si par mode on veut dire ensemble de temps régis et réservés à la subordination (cf. le coiuntivo italien). On parlera donc de temps au sens de tenses, chaque temps étant susceptible de renvoyer à un couple temps (time) / modalité à définir. La concordance des temps prend en breton un relief particulier dans le domaine qui serait en français celui du conditionnel et du subjonctif, en raison de l'existence dans les subordonnées de trois temps simples correspondant à trois niveaux d'irréalité : le futur/subjonctif, le potentiel et l'irréel, tous trois en usage actuel (à la différence du subjonctif imparfait français). - la confusion entre synchronie et diachronie dans la présentation de ces formes verbales dans les grammaires : en synchronie, le potentiel et l'irréel, utilisés en indépendantes et une partie de la subordination (conjonctives, relatives adverbiales, protases), se traduisent tantôt par des subjonctifs, tantôt par des conditionnels en français, et on peut décider de les nommer dans une partie des emplois conditionnels potentiel et irréel, comme le fait Hewitt 2010. Ceci est également justifié par la morphologie composite de ces temps (Bottineau 2010 et 2012) : de même que les conditionnels romans associent une marque de virtualité (l'infinitif en -r-) à une marque de passé (généralement l'imparfait, cf. -rait, mais le passé simple en italien, cf. rebbe), les morphèmes des deux conditionnels bretons sont des compositions d'éléments formateurs. Le potentiel en $-f e$, un ancien subjonctif passé, cumule un marqueur subjonctif $f$ et un marqueur imparfait $e$; l'irréel en -je, un ancien plus-que-parfait, cumule un ancien marqueur de passé simple $j$ et celui d'imparfait $e$. Pour cette raison, certaines grammaires (Trepos 2001) parlent de conditionnel-mode pour le premier et de conditionnel-temps pour le

\footnotetext{
${ }^{1}$ Nij 714 da Sydney, An Here, 2003, version bretonne des aventures de Tintin, Vol 714 pour Sidney, Casterman, 1963.

${ }^{2}$ http://makino.linguist.jussieu.fr/ARBRES/index.php/Bibliographie
} 
second, introduisant dans la description synchronique des considérations diachroniques qui placent la terminologie en porte-à-faux avec les emplois. La situation est encore plus illisible dans certaines syntaxes (Le Gléau 1973), qui oscillent entre les différentes terminologies en fonction des emplois : pour simplifier, la forme en - $f e$ est nommé potentiel en indépendante, futur indéterminé en cas de concordance et conditionnel-mode dans les phrases conditionnelles (dans le détail, la distribution terminologique est plus aléatoire que cela). Il en résulte que la description de l'emploi des temps dans les chapitres dédiés à la concordance fait parfois appel à des dénominations et classements ad hoc qui brouillent la lisibilité du rapport entre les faits particuliers et la cohérence générale de chaque temps.

- dans les différents secteurs de la grammaire, les approches schématiques du breton relèvent des faits de corrélation ou de concordance :

(a) selon les classiques, la phrase condionnelle devrait se former en utilisant deux temps identiques dans la protase et l'apodose, soit deux potentiels, soit deux irréels (à la manière des subjonctifs II de l'allemand). Les grammaires justifient cette prescription par la logique d'une part - il leur semble incohérent de corréler des niveaux différents d'irréalité - et par l'usage observé chez les auteurs classiques (non sans une certaine circularité, puisque nombre d'entre eux devait avoir connaissance des prescriptions des grammaires anciennes). L'usage parlé et écrit actuel dément cette règle, et on observe un décalage entre les exemples pris pour référence prescriptive et les pratiques ;

(b) dans les conjonctives, il existe des règles lexicales de rection modale, comparables à vouloir + subjonctif en français, qui devraient entraîner une concordance mécanique ; mais en breton la situation est complexifiée par l'existence du trio futur / potentiel / irréel (puisque le «futur » vaut également pour un subjonctif présent) et par une très large tendance à assouplir les faits de concordance sous l'effet de variations syntaxiques complémentaires comparables à je pense que + indicatif / je ne pense pas que + subjonctif en français.

La présente étude vise donc à montrer qu'il ne s'agit pas à proprement parler d'une concordance des temps (au sens de tenses en anglais) mais d'une concordance modale ou d'une harmonie des modalités entre la principale et la subordonnée, qui peuvent être mises en correspondance par des temps verbaux, mais également par des marqueurs discursifs plus diffus tels que la négation, certains adverbes, et la prosodie; et que cette harmonie des modalités fonctionne différemment dans des environnements présents et passés. Pour ce faire, on présente successivement la valeur intrinsèque des principaux temps concernés (section 1), les couplages et découplages modaux dans la phrase conditionnelle (section 2) et la pondération des concordances modale et temporelle dans les conjonctives (section 3 ).

\section{Les temps du breton hors faits de concordance}

Notre approche des temps verbaux est sous-tendue par la linguistique enactive (Bottineau 2012). Le principe général est que les formes langagières (morphèmes, lexique, constructions syntaxiques) n'encodent pas des schèmes représentationnels; ces formes constituent des comportements vocaux dont l'exécution suscite des effets perceptuels interprétables, lesquels correspondent à l'activation de processus cognitifs de structuration du sens. Les formes langagières sont utilisées soit pour «communiquer », faire parvenir autrui à un acte cognitifsémantique ciblé (parole extérieure, exophasie); soit pour «penser», se faire parvenir soimême à un acte de conscience complexe en simulant mentalement la vocalisation motrice des formes, leur perception associée, et les effets interprétatifs qu'elles activent (parole intérieure réflexive, endophasie). Un marqueur est donc défini du côté du signifiant comme une unité gestuelle d'activation et, du côté du signifié, un processus cognitif - interprétatif contribuant à l'économie générale de la production du sens au niveau de l'énoncé. La structure même du 
signifiant joue un rôle déterminant dans la définition de l'invariant opératoire du morphème dans son ensemble (ce qui fait de la linguistique enactive une linguistique du signifiant au sens de Letallec-Lloret 2010, 108), comme on va le voir pour la submorphologie de l'imparfait, du potentiel et de l'irréel.

Dans ce cadre, un temps verbal n'est pas le marqueur d'un repérage temporel de type chronologique, lequel est généralement explicité par les circonstants (Touratier 1996). Un temps verbal est un ensemble de morphèmes activant un processus cognitif déterminé qui, en relation aux autres éléments du contexte, contribue à inscrire un procès dans un espace mental compris comme imaginant le présent, le passé, l'avenir, le possible ou l'irréel. Le breton dispose des temps suivants: le présent, temps non morphématique, ne présente aucune instruction particulière, ce qui le fait coïncider par défaut avec l'actuel, au sens le plus large (ce qui est vrai ici et maintenant, ou ce qui est vrai en général), charge étant laissée au contexte de pointer un moment chronologique et une étendue au besoin ; la seule chose que le présent breton spécifie est lelocuteur comme auteur de la prédication (par opposition à une forme fréquentative périphrastique, qui renvoie le propos à une source énonciative collective, culturelle, proférant des vérités générales communes : e hañv e vez tomm an amzer «en été, il fait chaud »).

Tous les autres temps sont des inactuels au sens où leur morphologie active l'inscription du procès dans un espace mental correspond à un acte d'imagination non reliable à l'actuel, ce que sont le passé, le futur, le possible et l'irréel. Au sein de ce domaine de l'inactuel, on distingue le pseudo-actuel (l'inactuel conforme au vécu de personnel mémorisé ou au vécu imaginable et restituable) et le virtuel (l'inactuel non-conforme au vécu, typiquement le futur, le possible, l'irréel). Dans le champ du pseudo-actuel, le passé simple est utilisé par un locuteur qui à l'instant de parole proclame ce que le passé est, ce qui en fait un performatif utilisable par un auteur ayant autorité sur l'invention de son récit relativement à la communauté de destination : soit un historien face à la société, soit un enseignant face à une classe, soit un écrivain face à un lectorat. Cette fonction performative détermine les propriétés aspectuelles : la télicité, la couverture intégrale du procès si les bornes de l'évènement lexical le permettent (l'Aktionsart); le survol de tout le déroulement à l'instant de parole, qui crée un effet de sommaire ou de défilement accéléré; et la clôture terminale, qui permet la séquentialité narrative. Inversement, l'imparfait a pour effet de faire imaginer une situation sous un format conforme à du déjà vécu, qui peut être repérée en contexte comme passée ou imaginaire, structurellement conforme à l'expérience par son aspect : inaccomplie, pensée au rythme de défilement continu propre à l'actuel (la durée, la progression). La nonperformativité de l'imparfait en fait un médiatif : le locuteur qui l'utilise se présente comme le restaurateur d'une vision qui a été ou a pu être antérieurement produite par une source énonciative étrangère ; ce temps cumule deux voix énonciatives, celle d'un locuteur actuel qui relaie celle d'un énonciateur inactuel (Douay et Roulland 2012, 87, dans le cadre de la théorie de la relation interlocutive, parlent de duophonie pour l'imparfait et monophonie pour le passé simple). Ce dispositif diffère peu de celui du français, si ce n'est qu'on peut être surpris de la capacité de l'imparfait à faire imaginer une situation irréelle dans les protases alors même qu'il existe également un potentiel et un irréel.

Dans le champ du virtuel, la question posée par les temps est de déterminer si un évènement imaginaire sera ou non intégré à l'actuel, s'il est susceptible de se concrétiser. A cette question posée dans le cadre de l'interlocution, il existe trois réponses possibles en breton, activées par trois temps : «je suis sûr que oui, et je ne sollicite pas ton avis » (= le futur); «je ne sais pas, et je sollicite ton avis » (le potentiel) ; et « je suis sûr que non, et ne sollicite pas ton avis » (l'irréel). Ces trois temps correspondent ainsi à trois instructions hiérarchisées sur la question de l'actualisation du virtuel (oui, oui/non et non), dont deux univoques (le futur et l'irréel) qui impliquent une configuration de l'interlocution où le sujet parlant parle pour un 
couple locuteur/allocutaire indifférenciés, et un équivoque (le potentiel) qui implique une configuration de la relation interlocutive par laquelle le sujet parlant dissocie les points de vues possibles du locuteur et de l'allocutaire et ouvre un espace de négociation, mettant l'actualisation «en ballottage ». En articulant l'échelle des modalités avec les propositions de la théorie de la relation interlocutive (Douay et Roulland 2010), on peut pour le breton caractériser les correspondances comme suit.

Avec le futur/subjonctif, le locuteur arbitre seul, performativement et péremptoirement, que le virtuel doit s'actualiser. En relation avec un circonstant cela donne en français un « futur », et en relation avec une modalité telle que la volition, un «subjonctif », mais le geste cognitif sous-jacent est le même, dans sa dimension processuelle et interlocutive : Ne vo ket na ne vo 'ne sera pas ce qui ne sera pas' «pas question!»; Diskouez da fri ma welin anezhañ «montre ton nez que je le voie ». Le futur témoigne d'un geste d'arbitrage qui, selon les contextes, concerne un évènement relié à la ligne chronologique temporelle ou la cible d'une modalité, auquel cas il peut s'agir de modalité épistémique ou radicale : Ar c'hi, pa welo ur marc'h-houarn, a redo war e lerc'h en ur harzhal. 'Le chien, quand verra un cheval-fer, courra sur son arrière dans un aboyer.' "Quand le chien aperçoit un vélo, il le poursuit en aboyant. » vs Lavaro pezh a garo «qu'il dise ce qu'il voudra ».

Avec le potentiel, le locuteur indétermine son choix en matière d'actualisation et consulte implicitement l'allocutaire sur son propre point de vue : en indépendante $E$ c'hallfe bezañ ! « ça se pourrait ! », comme en subordonnée Lar dezhi gortoz betek ma 'm befe echuet « Dîteslui d'attendre que je sois arrivé », Aon 'm eus 'vefe re skuizh «j'ai peur qu'il ne soit trop fatigué », ce temps produit un effet d'indécision et de consultation interlocutive.

Avec l'irréel, le locuteur détermine un choix négatif catégorique ; il exclut le procès envisagé de toute possibilité d'actualisation et impose ce point de vue dans l'interlocution, le présentant comme la seule option envisageable sans possibilité de négociation : en indépendante Tennañ a rajec'h un olifant en un drepas! "vous tireriez un éléphant dans un couloir!» (comprendre: vous rateriez, le conditionnel irréel imposant le consensus sur la contrefactualité du procès imaginé, sans aucun fait de concordance) ; en subordonnée :

1. Evit kaout ur soñj eus ment Koskoriad an Heol e-keñver hini ar c'halaksienn, empennomp e vije hor galaksienn a-vent gant Frañs, da lavaret eo he dije $1000 \mathrm{~km}$ treuzkiz. Neuze e vije Koskoriad en Heol a-vent gant ur pezh ur santim euro. Gant ar binvioù modernañ zo ganimp evit beajiñ en egor, e vije ezhomm eus 15 vloaz d'un astraer da vont eus an Douar (kreiz hor pezh 1 santim euro) betek Ploudon (lezenn ar pezh). Evit tizhout ar steredenn dostañ d'an Heol (Alpha Centauri) war-hed 43 metr, ez istimer e vije ezhomm eus ur veaj 100000 bloaz., hag evit treuriñ ar c'halaksienn e vije ezhomm eus un hebeud miliardoù a vloavezhioù.

"Pour nous faire une idée de la taille du système solaire par rapport à celle de la galaxie, imaginons que notre galaxie soit de la taille de la France, c'est-à-dire qu'elle aurait $1000 \mathrm{~km}$ de diamètre. Alors notre Système Solaire serait de la taille d'une pièce d'un euro. Avec les moyens les plus modernes dont nous disposons pour voyager dans l'espace, il faudrait plus de 15 ans pour qu'un astronaute se rende de la Terre (le centre de notre pièce) à Pluton (le bord de la pièce). Pour atteindre l'étoile la plus proche du Soleil (Alpha Centauri) distante de 43 mètres, on estime qu'il faudrait un voyage de 100000 ans, et pour traverser la galaxie il faudrait plusieurs milliards d'années. »

Dans cet exemple, on trouve ensemble une occurrence subordonnée (le premier vije «soit») dont l'irréel (traduit par un subjonctif) est régi par empennomp «imaginons », ce qui est un fait de concordance de modalité (l'injonction à imaginer détermine un consensus sur 
l'irréalité) ; mais cette concordance initiale est ensuite reprise harmoniquement dans toutes les indépendantes (traduites par des conditionnels).

Les exemples qui précèdent montrent que ces trois temps, dans ou hors de toute corrélation de type «concordance », ont la possibilité de pondérer librement, en indépendante comme en subordonnée, la coordination interlocutive de l'acceptation ou du rejet de la perspective d'actualisation d'un évènement imaginé virtuellement. Compte tenu de ce dispositif, ces trois temps se prêtent à des engagements dans des jeux de corrélations et concordances temporelles remarquables.

\section{Les corrélations de temps verbaux dans les phrases conditionnelles}

Chacun des deux conditionnels est susceptible d'apparaître dans la protase ou dans l'apodose, et sera traduit en français par un imparfait ou un conditionnel selon la position qu'il occupe. Il ne s'agit donc pas d'une structure harmonique comparable à celles de l'allemand (qui associe le «subjonctif II » - le prétérit modal éventuellement appliqué à l'auxiliaire du futur werden issu du verbe signifiant «devenir »- dans la protase et l'apodose : wenn ich krank gewesen wäre, wäre ich zu Hause geblieben «si j'avais été malade, je serais resté à la maison »); il ne s'agit pas non plus d'une corrélation comparable à celle des langues romanes. La combinaison la plus fréquente et la moins marquée sémantiquement est celle qui associe l'irréel en protase initiale au potentiel en apodose finale : la position initiale de la protase thématise la condition en cohésion discursive, d'où une préférence pour l'irréel, lui-même fondé sur un consensus interlocutif ; la position finale de l'apodose rhématise la conséquence, d'où une préférence pour le potentiel, qui ouvre un débat interlocutif.

2. Ma ouijen skoazellañ e rafen n'eus forzh petra

'Si je savais épauler je ferais n'est force quoi'

«Si je pouvais aider, je ferais l'impossible.»

Mais cette indexation des temps (discussion interlocutive de l'actualisation) et des positions syntaxique (progression thématique / rhématique) n'est pas automatique : il existe trois autres combinaisons, avec le potentiel en protase si celle-ci n'est pas thématique et/ou avec l'irréel en apodose si celle-ci est thématique ou bloquée dans l'irréalis. L'interprétation dépend à la fois des temps associés et de la position de chaque volet de la phrase conditionnelle :

Protase / potentiel + apodose / potentiel :

3. Ya me a ve kontant da vervel en arme Ma c'hallfen soupsoniñ 'ves infidel din-me

« Oui moi je serais content de mourir à l'armée

Si je pouvais soupçonner que tu es infidèle avec moi »

4. Biskoaz c'hoaz! Marteze ne gavim ket e di, Jakez, hag e rankim distrei d'ar gêr gand bouzellou goullo? Ma ouifen kement-se, me a hourvezfe war an hent braz dioustu. "Peut-être que nous ne trouverons jamais sa maison, Jacques, et que nous resterons hors de la ville et l'estomac vide ? Si je le savais [=Si j'en avais la certitude], je me coucherais en plein milieu du chemin. »

Protase / irréel + apodose / irréel : 
5. Ma c'halljen mont ez ajen

«si je pouvais y aller, j'irais »

(mais il va de soi que ce ne sera pas le cas)

Protase / potentiel + apodose / irréel :

6. Ha pa zistrofe ar gouloù, e vije reziwezhat.

'et quand [bien même] reviendrait la lumière, ce serait trop tard'

«Même si la lumière revenait, ce serait trop tard. »

(protase : hypothèse négociée; apodose : conséquence admise comme allant de soi pour les interlocuteurs)

On retrouve la même liberté combinatoire avec les formes composées :

Potentiel + potentiel

7. En em zigareziñ a rafen ma 'm befe lavaret traoù drouk pe direizh, met aze ne welan ket tamm ebet evit petra e rankfen en em zigareziñ, n'eus netra tagus...

«Je m'excuserais si j'avais dit du mal ou des choses injustes, mais là je ne vois absolument pas pourquoi je devrais m'excuser, il n'y a rien de méchant... »

8. Desket ' $m$ eus dit bezañ ur gwir aerouant... Ha graet ' $m$ befe se ma' $m$ befe bet c'hoant da ober ac'hanout ul loen doñv dizañjer?

«Je t'ai appris à être un véritable dragon... Est-ce que je l'aurais fait si j'avais eu

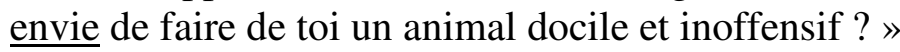

Irréel + irréel

9. M'am bije labouret em bije arhant.

«Si j'avais travaillé, j'aurais de l'argent. »

Irréel + imparfait

Cette combinaison, plus courante en breton qu'en français, est signalée parmi les cas de concordance « surprenante » sur le site ARBRES ${ }^{3}$ :

10. Ma 'm-bije lonket ar boulou, oa greet ganen!...

«Si j'avais avalé les boules, c'en était fait de moi !»

Dans cette combinaison, la protase impose un consensus sur l'inactualisabilité du virtuel envisagé. L'apodose pourrait rééditer cette démarche et être mise à l'irréel elle aussi, mais l'objectif du locuteur est au contraire de faire imaginer à l'allocutaire ce qu'il aurait vécu au cas où la transition condition / conséquence se serait réalisée. L'utilisation de l'imparfait suscite l'inscription de l'évènement dans un espace mental correspondant à un pseudo-actuel qui n'est pas un passé, il s'agit bien d'une fiction, mais son contenu est analogue à celui d'un vécu remémoré. L'effet produit consiste d'une part à souligner le caractère immanquable de l'enchaînement causal, et d'autre part à faire éprouver par empathie la teneur de l'expérience simulée. Il faut bien voir que l'imparfait, le potentiel et l'irréel ont en commun le marqueur $e$,

\footnotetext{
${ }^{3}$ http://makino.linguist.jussieu.fr/ARBRES/index.php/Concordance_des_temps
} 
dont la valeur instructionnelle est l'activation d'une simulation imaginaire. Pour l'imparfait, cette simulation seule ne dit pas si elle s'applique à un évènement réel ou irréel, charge étant laissée au contexte de le spécifier, comme ici la présence d'une condition dans une protase. Pour le potentiel et l'irréel, l'alternance $f / j$ correspond à deux instructions interprétatives complémentaires qui concernent le débat relatif à l'actualisabilité de la simulation, débattue et ouverte avec $f$, fermée et non discutée avec $j$. Le simple fait d'ouvrir ce débat sur l'actualisabilité par l'alternance $\mathrm{f} / \mathrm{j}$ fait automatiquement que l'imparfait e de simulation renvoie à un évènement virtuel : cette alternance joue à l'intérieur de la composition morphologique le même rôle spécification que le contexte à l'extérieur de la forme d'imparfait. On comprend que ces trois temps sont harmonisés par la présence de l'élément formateur commun $e$, opérateur de simulation qui établit une concordance modale entre protase et apodose quelle que soit la configuration retenue - si l'on veut bien accepter l'idée d'une concordance submorphologique à un niveau aussi élémentaire que celui des éléments formateurs.

$\mathrm{Au}$ niveau morphologique, de toutes ces fluctuations, Chalm $(2008,73)$ conclut que «la concordance des temps n'est pas obligatoire» dans les phrases conditionnelles. Cette formulation est déconcertante parce qu'elle assimile deux niveaux distincts. Il est exact qu'il n'y a pas obligation d'harmonisation interlocutive : une protase peut recevoir un virtuel rejeté (irréel) et l'apodose un virtuel discuté (potentiel), et inversement. Mais il existe bien des phénomènes de concordance incontournable : avec une protase au présent, l'apodose peut être au présent ou au futur, rarement au potentiel, jamais à l'irréel. Avec une protase au potentiel ou à l'irréel, il est exclu que l'apodose soit au présent ou au futur. La tendance générale va à la discrimination de deux niveaux énonciatifs : le présent dans la protase valide l'hypothèse comme un actuel assumé comme tel par le locuteur à l'instant de parole, alors que l'emploi de l'un des «conditionnels » (potentiel ou irréel) fait de l'hypothèse un inactuel que le locuteur renvoie pour la forme à une source énonciative altérisée, ce qui lui permet de la mettre en perspective, la soumettre au regard interlocutif et engager la négociation pondératrice. La trace morphologique de cette mise à distance réside dans le $e$ de $f e$ et $j e$, élément formateur commun aux conditionnels et morphème de l'imparfait, indice de la médiativité de ces trois temps. Il se dégage ainsi un schéma général de concordance énonciative à deux niveaux : les couples protase / apodose énonciativement non distants (sans e) attribuent au locuteur la prise en charge de la transition condition / conséquence; les couples protase / apodose énonciativement distants (avec e) attribuent cette transition à un énonciateur autre que le locuteur pour la forme, afin de placer le locuteur en position d'observateur / évaluateur de la relation, d'où l'effet sémantique et pragmatique de prise de recul et de réflexion (au propre et au figuré). Et à ce second niveau, il subsiste une marge de manœuvre pour des ajustements interlocutifs de la modalité : dans les apodoses, l'imparfait, le potentiel et l'irréel correspondent respectivement aux configurations 0,1 et 2 de la théorie de la relation interlocutive, à savoir, respectivement, pas de prise en charge interlocutive de la simulation, divergence interlocutive sur son (in)actualisabilité, et convergence sur son inactualisabilité. Ces ajustements vont être constitutifs du jeu complexe de la concordance dans les interrogatives et les conjonctives.

\section{De la concordance des temps à la concordance modale}

\subsection{Le verbe de la principale est au présent}

Dans les conjonctives, le temps du verbe régi résulte d'un compromis entre la visée modale issue du verbe recteur et le format de la relation interlocutive recherché : la certitude et la volition catégorique appellent le futur et l'absence de compromission. 
11. Gouzout a ran e teuio.

savoir je-fais que il-viendra

« Je sais qu'il viendra »

Avec diwall ma « veiller à ce que » :

12. Ho tever eo diwall ma vo komzet mat ha flour ar brezhoneg.

« Votre devoir est de veiller à ce que l'on parle un breton correct et fluide. »

13. Pennlabour an DGLF eo diwall ma vo doujet ouzh lezenn ar 4 a viz Eost 1994, anvet ivez lezenn Toubon. Kemer a ra perzh ivez e gallekaat an dud enbroet.

La mission principale de la DGLF est de veiller à ce que soit respectée la loi du 4 août 1994, également nommée loi Toubon.

14. Diwall ma chomo ar muia lêz posubl e-barz.

Attention à ce qu'il reste le plus de lait possible à l'intérieur.

D'autres verbes et expressions régissent le potentiel; la visée modale n’implique pas catégoriquement l'actualisation du virtuel et ouvre le débat interlocutif :

15. C'hoant am eus ma rofec'h din an aotre.

J'ai envie que vous me donniez la permission.

Mais après de nombreux verbes, on observe une alternance futur / potentiel selon que le locuteur impose une actualisation ou ouvre un espace de discussion à l'allocutaire. Avec goulenn « demander » :

16. Goulenn a ran e teuio.

« Je demande / j'exige qu'il vienne"

(non négociable)

17. Goulenn a ran e teufe

« Je demande à ce qu'il vienne »

(négociable)

Avec ret eo « il faut que », «il est nécessaire que »:

+ futur :

18. Ret eo ma vo digoret frank dor ar skolioù d'ar brezhoneg, keneve-se e vo kollet hor yezh un deiz bennak.

«Il faut que les portes des écoles soient grandes ouvertes au breton, sinon un jour notre langue sera perdue. »

+ potentiel :

19. Ret eo ma ve kollet ho penn da gaout c'hoant da werzhañ ho marc'h evit kaout arc'hant da reiñ adarre d'ar beorien.

«Il faut croire que vous avez perdu la tête pour avoir envie de vendre votre cheval pour trouver de l'argent et le redonner aux pauvres. » 
De la même manière, les subordonnées de but introduites par evit ma sont suivies du futur si le locuteur est déterminé, du potentiel s'il est prêt à négocier :

+ futur :

20. Evit ma c'hallo an holl dud kaout ul lojeiz ekologel hag arboellus 'pour que puisse tout le monde avoir un logement écologique et qui consomme peu' « Pour un habitat économe, écologique et accessible à tous » (slogan)

21. Stourmomp evit ma vo ofisiel ar brezhoneg e Breizh a bezh!

«Luttons pour que le breton soit officiel dans toute la Bretagne!»

+ potentiel :

22. Ya, siwaz. A-wechou em-eus c'hoant lakaad eur mên e-kreiz ar pilerad amann evid ma pouezfe muioh. (Pêr Jakez-Helias)

«Oui, malheureusement. Parfois j'ai envie de mettre une pierre au milieu de la motte pour que ça pèse plus lourd. »

Le principe est que le verbe régi est en accord interlocutif avec la visée modale prêtée par le locuteur au verbe de la principale. Certains verbes se prêtent à des visées divergentes, clairement soulignées par la situation ou la prosodie, mais non explicitées directement au niveau de l'unité lexicale, comme supra le verbe goulenn, dont le sens est « réclamer» (avec détermination) ou «demander» (avec ouverture) selon la manière de le présenter. Le plus souvent, la force de la visée modale est explicitée par un élément du contexte, comme le nom c'hoant «envie » dans l'exemple qui précède. Une négation au niveau de la principale ou du subordonnant affaiblit la visée, compromet la détermination du locuteur et appelle le potentiel en lieu et place du futur, comme en français on trouve le subjonctif après je ne pense / crois pas que, par exemple me a gred e raio glav «je crois qu'il pleuvra » vs ne gredan ket e rafe glav « je ne crois pas qu'il pleuve ».

Avec diwall :

23. Diwall na vefes gwelet!

«Fais attention à ne pas être vu!» (cela pourrait arriver)

24. Diwallit na vefe disrannoù en ho touez.

'Attention à ce qu'il n'y ait pas de séparation dans votre mélange'

« Faites attention à ce que votre mélange soit homogène. » (cela peut se produire)

Le futur peut être utilisé, de manière nettement plus marquée, pour maintenir la force de la visée modale du locuteur. Ceci se produit dans tous les contextes où il est évident que le locuteur lui-même redoute la survenue de l'évènement proscrit :

25. Diwall na lazhi den ebet.

'veille à ce que tu ne tues personne'

«Prends garde à ne tuer personne». 
En breton parlé, cette nuance se traduit souvent par le découplage du conjonctif négatif $n a$ en conjonctif $m a+$ négation $n e$ :

\section{Diwall ma ne vo ket kousiet ar vugale.}

«Attention à ce que les enfants ne soient pas sales. »

Ce type d'emploi du futur après diwall après une négation en lieu et place du potentiel attendu est favorisé par la présence d'une modalité de renforcement telle que ret eo « il faut »:

27. Evit ar blezad kentañ en em gaver get un toull : $20 €$. N'eo ket pikol tra, met ret eo hel lâret ha diwall ma ne vrasao ket en amzer da zonet.

«Pour la première année nous nous retrouvons avec un déficit de $20 €$. Ce n'est pas important mais il faut faire attention à ce que cela n'augmente pas. »

Les alternances qui précèdent manifestent le système suivant : le verbe d'une principale régit le futur ou le potentiel en fonction de la visée modale qu'il projette et de la configuration interlocutive qui en résulte, à savoir que l'actualisation du procès virtuel est soit décrétée par le locuteur seul (futur), soit débattue avec l'allocutaire (potentiel). De nombreux verbes se prêtent aux deux types de visée, soit parce qu'ils sont polysémiques (goulenn: demander / exiger), soit parce que leur sens est pondérable par des éléments contextuels. Parmi ces éléments cohabitent d'une part des indicateurs d'affaiblissement modal tels que la négation, l'interrogation, des adverbes hypothétiques comme morse "peut-être », et d'autre part des indicateurs de renforcement modal tels que l'impératif, la prosodie injonctive, ou des expressions de jugement catégorique telles que ret eo «il faut». Le modèle standard de rection du verbe principal peut ainsi être revu dans le sens du renforcement ou de l'affaiblissement en fonction des éléments discursifs environnants qui modulent sa visée modale : un verbe normalement suivi du potentiel sera suivi du futur s'il est lui-même à l'impératif ou précédé de ret eo ; un verbe normalement suivi du futur sera accompagné du potentiel sous l'effet d'une négation ou d'un quelconque autre atténuateur.

Ainsi, au présent, et indépendemment de toute variation du temps du verbe principal par l'emploi du passé simple ou de l'imparfait, on observe un mécanisme de concordance modale par laquelle le temps de la subordonnée (futur ou potentiel) dépend de la visée modale du verbe principal telle qu'elle a été modulée par les indicateurs lexicaux, morphosyntaxiques et prosodiques qui l'environnent. Cette concordance modale est une version élargie d'un phénomène plus localisé que Le Gléau nomme l'attraction modale :

« Une principale au futur ou à l'impératif ou à l'optatif futur entraîne souvent un verbe subordonné lui-même au futur, alors que le sens du verbe ne l'exige pas. On peut parler d'attraction modale. » (Le Gléau 1999, 130)

Mais il s'empresse de préciser :

«Liberté de choix de l'écrivain, influence de la courtoisie et surtout tempérament de chacun : souplesse dans le Léon, fermeté dans le Vannetais. Dans l'attraction modale, on nage dans le flou des nuances et des interprétations. »

Il n'est pas surprenant que ce phénomène de nature modale présente une certaine souplesse. Toutefois, la prudence de Le Gléau s'explique aussi par le fait qu'il a focalisé l'attraction modale sur le temps du verbe principal sans toujours expliciter l'influence des autres 
paramètres. La concordance modale joue dans les deux sens (renforcement et affaiblissement), et elle peut mettre en jeu des indicateurs conflictuels. Dans l'exemple supra ret eo hel lâret ha diwall ma ne vrasao ket en amzer da zonet « il faut faire attention à ce que cela n'augmente pas », diwall, suivi du futur, devrait être ici suivie du potentiel à cause de la négation affaiblissante (faisons attention à ce que cela ne se produise pas : cela pourrait arriver), mais celle-ci est neutralisée par ret eo «il faut », qui joue un rôle de renforcement et restaure la visée catégorique, faisant pression pour que la chose n'arrive pas. Dès lors que l'on introduit cette notion de visée modale, et qu'on la pondère en tenant compte non seulement du sens du verbe lexical, mais également et surtout des indicateurs contextuels qui le modulent, on s'aperçoit que les fluctuations qui donnent au système un comportement à l'allure chaotique sont en réalité sous-tendues par un dispositif précis de régulation. Un exemple intéressant est le cas de me 'gavfe "j'aimerais / je voudrais » (que), qui selon Le Gléau est normalement suivi du potentiel, conformément à l'affaiblissement de la visée par le potentiel du verbe de la principale.

28. Me garfe ma vefec'h dinec'h e-keñver traoù an douar.

«J'aimerais que vous soyez détendu en ce qui concerne les choses de la terre. »

Mais l'usage montre que les locuteurs appliquent à la subordonnée le niveau de modalité qui correspond à leur intention réelle; on peut déguiser en sollicitation mesurée ce qui est en rélité une exigence ferme, comme dans :

29. Me garfe ma ne vo ket troet "guerre d'édition" gant "brezel embannerezh" 'moi aimerait que ne soit pas traduit 'guerre d'édition' par 'brezel embannerezh' « J'aimerais qu'on ne traduise pas 'guerre d'édition' par 'brezel embannerezh' »

où l'auxiliaire du verbe passif de la subordonnée, vo, et au futur/subjonctif à valeur injonctive et non au potentiel vefe comme le laisserait attendre une approche déterministe de la concordance. Dans cet exemple, vo est en accord modal non pas avec la modalité de surface marquée par garfe, mais avec la modalité intentionnelle réelle qui sous-tend la déclaration. Considérons ce que devient ce dispositif dans un environnement passé.

\subsection{Le verbe de la principale est au passé}

Le breton classique collecté auprès des auteurs littéraires par les principales grammaires descriptives et normatives ne mentionnent qu'un usage : le curieux «irréel de concordance », qui apparaît dans la subordonnée si le verbe de la principale est à l'imparfait ou au passé simple (mais pas au passé composé, à cause du temps présent de l'auxiliaire) : Gouzout a raen e teuje «je savais qu'il viendrait », Ne lavare ket pegoulz e vije deut «il ne disait pas quand il viendrait », Ne greden ket e raje glav «je ne pensais pas qu'il pleuvrait » (Favereau 1997, 333), sans alternance modale autour du rapport affirmation / négation. Cette construction inattendue est la seule où l'irréel survient manifestement à "contretemps » par rapport à sa valeur centrale de consensus sur l'inactualisabilité d'un procès virtuel; elle perturbe remarquablement les descriptions : Favereau parle, non sans hésitation, d'une «sorte de passé du futur-subjonctif »; le Gléau $(1999,1333)$ rapproche cet emploi de l'irréel de celui du subjonctif grec ancien, alors que le potentiel serait analogue au subjonctif imparfait latin. La situation s'opacifie encore lorsque l'on observe les formes composées au parfait et les traductions proposées, comme dans ces exemples de Chalm 2008 :

30. Kredet em eus en defe kanet.

« J'ai cru qu'il aurait chanté. » (traduction de la grammaire de Chalm 2008) 


\section{Kredet em boa en dije kanet.}

«J'avais cru qu'il aurait chanté ».

Cependant, en breton actuel le potentiel gagne du terrain au détriment de l'irréel, y compris dans les structures à concordance des temps, comme le signale Le Gléau :

32. Kinnig a ris ober ur stagadenn vrezhonek gant ma paefe din un nebeut santimoù. 'offrir fis-je faire une annexe breton avec si paie à-moi un peu centimes'.

«Je me proposai pour rédiger une annexe en breton moyennant une petite rémunération. »

33. E 1851 e voe goulennet gant bodadeg an dileuridi ma vefe kelennet ar flandrezeg. 'En 1851 fut demandé par l'assemblée des représentants que soit fût enseigné le flamand.'

«En 1851, l'assemblée des représentants demanda à ce que le flamand soit / fût enseigné. »

$\mathrm{Au}$ lieu de nous focaliser sur l'incohérence apparente des valeurs d'emploi des temps, considérons les choses du point de vue du système d'oppositions. Dans l'usage classique, l'emploi exclusif de l'irréel de concordance après un verbe au passé neutralise toute possibilité d'alternance entre différents temps verbaux, quels que soient les autres paramétrages contextuels du verbe de la principale (affaiblissement par la négation ou renforcement par divers adverbes et constructions) : autrement dit, la concordance modale qui est la règle au présent est remplacée purement et simplement par une concordance temporelle au passé, les deux s'excluant mutuellement. D'un point de vue sémantique et énonciatif, l'implication est que dans ce système ancien, la pondération de la visée modale impriméepar le locuteur n'est réalisée que si le verbe est au présent, qui se trouve être le temps de la prise en charge énonciative des actes de modalisation et le temps par lequel se régulent les configurations de la relation interlocutive et de la réception par l'allocutaire. Dès lors que le verbe est au passé, la visée modale n'est plus attribuée au locuteur comme source présente, mais à un énonciateur distancié, éventuellement altérisé, ce qui suspend l'effet de la modalisation et explique le verrouillage modal. Concrètement, dans gouzout a raen e teuje « je savais qu'il viendrait », l'irréel ne vaut pas pour ce qu'il semble signifier en lui-même, l'irréalité de la venue, mais pour la manière dont il s'oppose aux deux autres temps : le futur impliquerait une visée actualisatrice catégorique présente; le potentiel, une visée actualisatrice en discussion; et de ce fait, l'irréel, une absence de visée tout court du fait de l'ancrage passé du verbe principal, décroché de l'instant présent d'énonciation et d'interlocution. Cet emploi de l'irréel signe le fait que le locuteur ne s'identifie pas à l'auteur de la visée et ne participe pas à son actualisation, indépendemment du fait que l'évènement est réel, ce qu'indique le sens du verbe savoir.

Dans les emplois plus récents signalés par Le Gléau et que l'on retrouve dans le breton parlé sur divers forums, la situation est différente. D'une part, l'emploi du potentiel après un verbe au passé fait du passé un marqueur de l'abaissement du niveau de visée modale au même titre que la négation : le temps du verbe lexical de langue et les marqueurs discursifs de la modalité entrent dans le même paradigme, unifiant le traitement des concordances modale et temporelle qui étaient disjoints et opposés dans le modèle ancien. D'autre part, l'introduction du potentiel dans cette position vient compléter celui de l'irréel préexistant, et non le remplacer, en sorte qu'il apparaît une alternance : l'alternance futur / potentiel du verbe régie après un verbe recteur au présent devient une alternance potentiel / irréel du verbe régi après 
un verbe recteur à un temps du passé. On distingue ainsi des emplois comme Ne greden ket $e$ c'hallje mont-en-dro «je ne croyais pas qu'il pourrait revenir» et Den ebet ne grede $e$ c'hallfe mont en-dro "Personne ne s'imaginait qu'il pourrait revenir », où l'emploi du potentiel en lieu et place de l'irréel laisse entendre ce qui se vérifie effectivement en contexte : que «il » est effectivement revenu, et que le locuteur, en utilisant le potentiel, a laissée ouverte la perspective de l'actualisabilité en vertu de ce que lui-même sait, envers et contre le point de vue général évoqué dans la principale, ce que restitue ma traduction par le verbe «s'imaginer ». Schématiquement, il en résulte un système parallèle : au présent, me $a$ gred e raio glav (affirmation + futur) «je crois qu'il pleuvra » vs Ne gredan ket e rafe glav (négation + potentiel) 《je ne crois pas qu'il pleuve»; au passé, me a grede e rafe glav (affirmation + potentiel) « je croyais qu'il pleuvrait » vs ne gredan ket e raje glav (négation + irréel) «je ne croyais pas qu'il pleuvrait». Mais ces configurations ne sont pas figées, puisque le rang du sujet grammatical joue également un rôle important: Den ne grede e rafe glav (potentiel malgré la négation) - dès lors que le sujet est distinct du locuteur, celui-ci peut se permettre de distinguer son prore point de vue de celui prêté au sujet grammatical en jouant sur une rupture de la concordance modale.

Il arrive même que la concordance temporelle au passé ouvre des possibilités d'alternance modale qui ne se présentent pas au présent. L'expression spi am eus 'espoir mien est' «j'espère que » est suivie du futur au présent, ce qui est logique compte tenu du sens ; les occurrences fournies par google se comptent par milliers, et ne sont apparemment jamais accompagnées d'un atténuateur qui provoque le remplacement du futur par le potentiel :

34. Spi am eus e teuoc'h a-benn da grouiñ ur skol divyezhek gallek-okitanek. « J'espère que vous parviendrez à créer une école bilingue français-occitan. »

35. Spi am boa koulskoude e vefe klevet komz diouto kalz nebeutoc'h dre ma oant null «J'espérais pourtant qu'on entendrait beaucoup moins parler d'eux vu qu'ils étaient nuls »

36. Spi am-eus o-po c'hoant kana asamblez ganeom ivez bremaig.

« J'espère que vous aurez bientôt envie de chanter avec nous aussi. »

37. Spi am eus e vo posubl deoc'h degas ar profoù din!

« J'espère qu'il sera possible d'emporter les épreuves avec moi !»

Ces emplois produisent l'effet d'une réminiscence, d'un locuteur qui se souvient actuellement d'un état psychologique antérieur, s'éloignant par là même de l'allocutaire, qui n'a pas accès à cette expérience.

Tout change au passé avec spi am boa «j'espérais que ». Dans la littérature classique, cette structure aurait été obligatoirement suivie de l'irréel. En breton parlé actuel, on observe l'alternance potentiel / irréel. Le potentiel est utilisé lorsque le locuteur maintient, malgré l'emploi du passé dans la principale, le point de vue de l'expérient qui attend encore de savoir si la chose espérée se réalise, comme dans cet extrait de heroic fantasy:

38. C'hoant ' $m$ boa, spi ' $m$ boa e selaoufent ac'hanon o welet e oan mignonez gant un aerouant.

« Je voulais, j'espérais qu'ils m'écoutent en voyant que j'étais l'amie d'un dragon... »

39. Buan e oa aet evit ma breur e Brest ha spi am boa e vefe ken aes...

« J'étais vite allé voir mon frère à Brest et j'espérais que ce serait aussi facile... » 
Ces emplois du potentiel produisent l'effet d'une réminiscence, d'un locuteur qui se souvient actuellement d'un état psychologique antérieur, s'éloignant par là même de l'allocutaire, qui n'a pas accès à cette expérience. L'emploi de l'irréel porte au contraire l'attention sur le point de vue du locuteur présent telle qu'il se présente actuellement, distinct de celui qui a vécu un espoir dans le passé, dans sa relation à l'allocutaire par l'acte de langage, et qui n'est plus en position d'espérer la réalisation de l'évènement, qu'il y ait eu succès ou non :

40. Spi am boa ez pije gounezet ar priz.

«J'espérais que j'aurais gagné le prix. »

La concordance modale joue manifestement sur le niveau énonciatif ouvert par le submorphème d'imparfait $e$, et l'alternance potentiel irréel permet de jouer sur le centrage élocutif ou interlocutif du point de vue - le potentiel focalisant un état passé du locuteur, non partagé dans le dialogue, et l'irréel un état présent des interlocuteurs associés, partageant un regard fédérateur, une source commune de visée modale relative au procès. Ce point est important, il illustre l'erreur qu'il y a à s'imaginer que les opérations langagières seraient exclusivement centrées sur le sujet parlant. Si le sujet parlant est un être corporel vivant, produisant apparemment des messages du seul fait de son comportement d'agent observable autonome et discret, le locuteur est un rôle abstrait qui se définit dans et par l'interlocution et se formalise relationnellement par des alternances morphémiques ad hoc, comme le veut la relation interlocutive. Et du point de vue de la grammaire enactive, tout acte de production d'un signifiant par une boucle motri-sensorlelle produit sur les récepteurs, les sujets parlant et écoutant, un effet intersubjectivement distribué qui consiste à agir sur la relation interlocutive et la reconfigurer selon un format donné relativement à une question ciblée, qu'elle aussi élémentaire que la relation discursive ou indexicale à un objet (articles, démonstratifs) ou la relation modale à un procès virtuel (potentiel, irréel); l'effet est de coordonner, par cette action morphématique, les points de vues confrontés en termes d'association ou de dissociation. La relation entre théorie de la relation interlocutive et grammaire enactive demanderait à être explicitée en invoquant le paradigme de la « cognition des 4E » (embodied « incarnée », embedded «située», enactive «enactive» et extended «étendue », ce qui ne peut être fait ici ; on retient simplement le fait que l'ouverture d'alternances modales en contexte passé en breton s'explique dans le cadre de cette problématique.

\section{Conclusion}

Les faits décrits suggèrent qu'en breton il existe deux phénomènes distincts : au présent, une concordance modale par laquelle la relation verbe régissant - verbe régi se module par divers marqueurs syntagmatiques, lexicaux, discursifs et prosodiques, qui jouent dans le sens d'un relèvement ou d'un rabaissement de la force de visée actualisatrice et d'une régulation de la relation interlocutive relative à cette visée. Au passé, en breton littéraire classique, il se présente une concordance des temps mécanique, disjointe de la première, qui la neutralise pour cause de mise à distance énonciative et virtualisation de la source de la visée. En breton actuel, on observe une dynamique d'intégration des deux types de concordances : d'une part, le marquage passé du verbe principal peut produire sur le verbe régi des effets d'accord temporel analogues à ceux créés par la négation; d'autre part, l'émergence d'une alternance potentiel / irréel en contexte passé permet d'y introduire les alternances de concordance modale qui dans le système classique ne jouent qu'en contexte présent. Cet enrichissement permet en outre l'apparition de faits de désaccord modal en environnement passé, par lesquels un locuteur peut faire entrevoir un point de vue différent de celui du sujet grammatical. Ce 
dispositif élaboré, très riche en nuances, est rendu possible par l'épaisseur systémique du domaine de l'inactuel avec trois temps simples, et l'inexistence de la distinction indicatif / subjonctif en tant que modes syntaxiques en breton, puisque tous les temps concernés s'utilisent en indépendante comme en subordonnée, acquérant dans le premier domaine des valeurs systémiques qu'elles recyclent dans le contexte des relations articulées du second (phrases conditionnelles et surbordination avec concordances). Le breton parvient ainsi à systématiser des tendances que l'on observe de manière plus sporadique dans les langues romanes.

\section{Bibliographie}

Ar Merser A. (1990), Précis de grammaire bretonne, Emgleo Breiz, Ar Skol Vrezoneg, Brest.

BAKHTINE M. ([1929] 1977), Marxisme et philosophie du langage, Minuit, 1977.

LE BESCO P. (1997), Parlons breton, langue et culture, L'Harmattan, Paris.

LE BESCO P. (2005), Le breton de Belle-Île en Mer, Brud Nevez.

BotTinEAU D. (2006), «La morphosyntaxe allocutive du sens grammatical », Revue de Sémantique et Pragmatique 19/20, juin-décembre 2006, 93-120.

BotTineau D. (2010), «Les temps du verbe breton : Temps, aspect, modalité, interlocution, cognition - des faits empiriques aux orientations théoriques », C. Douay (éd.), Système et chronologie, Rennes : Presses Universitaires de Rennes, 129-157.

Bottineau D. (2010), «Language and enaction », J. Stewart, O. Gapenne, E. Di Paolo (eds), Enaction: toward a new paradigm for cognitive science, MIT, 267-306.

BotTineAU D. (2011), «Parole, corporéité, individu et société : l'embodiment entre le représentationnalisme et la cognition incarnée, distribuée, biosémiotique et enactive dans les linguistiques cognitives », Guignard J.-B. (dir), Linguistique cognitive : une exploration critique, Intellectica 56, 2011/2, 187-220.

BotTineau D. (2012), «Syntaxe et interlocution en français et en breton », C. Douay \& D. Roulland (dir.). L'interlocution comme paramètre, Presses Universitaires de Rennes, 183206.

BotTineAu D. (2012), «La parole comme technique cognitive incarnée et sociale », Linguistique et phénoménologie du langage, La Tribune Internationale des Langues Vivantes, 52-53, Perros-Guirec : Anagrammes, 44-55.

BotTineAU D. (2012), «Les conditionnels potentiel et irréel en breton : De la morphologie temporelle à la valeur instructionnelle », J. Brès, S. Sarrazin, S. Azzopardi (dir.), Faits de langues 40, Ultériorité dans le passé: le conditionnel, Bern, Peter Lang, (à paraître: décembre 2012).

BOURDIN Ph. (1996), «La concordance des temps aux confins de l'accord? », Faits de langues, 8, 207-216.

CARrasco Gutiérrez Á. (2000), La concordancia de tiempos, Madrid, Arco/Libros.

CARrer Ph. et alii (1986), Permanence de la langue bretonne, de la linguistique à la psychanalyse, Institut Culturel de Bretagne / Skol Uhel ar Vro, Rennes.

Chalm E. (2008), La grammaire bretonne pour tous, An Alarc'h Embannadurioù / Editions Le Cygne, Lannion.

COURSIL J. (2000), La fonction muette du langage, Ibis Rouge Éditions, presses Universitaires Créoles, Guadeloupe.

Desbordes Y. (1999), Petite grammaire du breton moderne, $4^{e} e ́ d$., Mouladurioù hor yezh, Lesneven. 
DENEZ P. (1986), «Contraintes formelles de la structuration de la phrase bretonne », in CARRER, Philippe et alii (1986), Permanence de la langue bretonne, de la linguistique à la psychanalyse, Institut Culturel de Bretagne / Skol Uhel ar Vro, Rennes, 19-44.

DouAY C. (2000), Eléments pour une théorie de l'interlocution, Un autre regard sur la grammaire anglaise, Presses Universitaires de Rennes.

Douay C. \& Roulland D. (2012), «L'interlocution comme clé du contrastif », C. Douay \& D. Roulland (dir.). L'interlocution comme paramètre, Presses Universitaires de Rennes, 77-94.

LE DÛ J. (1990), « The semantics of the simple tenses of the verb at Plougrescant », Ann T.E. Matonis \& Daniel F. Melia (eds), Celtic Language, Celtic Culture, Ford and Baile, Van Nuys, California.

FAVE V. (1998), Notennou yezadur. Emgleo Breiz: Brest. (« Notes de grammaire »)

FAVEREAU F. (1997), Grammaire du breton contemporain, Skol Vreizh, Morlaix.

LE GLEAU R. (1973), Syntaxe du breton moderne (1710 - 1972), Editions La Baule.

LE GLEAU R. (1999), Etudes syntaxiques bretonnes; 4 vol., René Le Gléau (ed.), Brest.

HEINECKE J. (1999), Temporal Deixis in Welsh and Breton. Heidelberg: Carl Winter.

HEMON R. ([1940] 1975), Grammaire bretonne, $8^{\mathrm{e}}$ éd., Al Liamm, Brest.

HewitT S. (2010), «Mood in Breton », B. Rothstein \& R. Thieroff (eds), Mood in the Languages of Europe, Benjamins, 292-308.

Humphreys, H. L. (1995), Phonologie et morphosyntaxe du parler breton de Bothoa en Saint-Nicolas-du-Pélem (Côtes d'Armor), Ar Skol Vrezoneg - Emgleo Breiz, Brest.

KeRVELla F. ([1947] 1995), Yezhadur bras ar brezhoneg, Al Liamm. ("Grande grammaire du breton")

LECLERC, Abbé (1911), Grammaire bretonne du dialecte de Tréguier, Editions Prud'homme, Saint-Brieuc.

LE TALLEC-LLORET Gabrielle (2010), La concordance des temps en espagnol moderne, unité du signe, modes, subordination. Presses Universitaires de Rennes.

LuQuet G. (2010), «La concordance des «temps»? Soit. Mais des temps de quels modes ? », in G. Luquet (éd), La concordance des temps. Moyen-Âge et époque moderne, Actes du colloque sur la concordance des temps, SEMH-Sorbonne - GERLHIS, Colegio de España, Paris, 30-31 mai 2008, 83-93.

OGIEN A. (2007), Les formes sociales de la pensée, La sociologie après Wittgenstein, Colin.

REID L. A. 1971. "Tense Sequence in Procedural Discourse." The Archive 2.15-42

Roulland D. (2010), «Temps et théorie des systèmes », C. Douay (éd.), Système et chronologie, Rennes : Presses Universitaires de Rennes, 71-90.

Trepos P. (2001), Grammaire bretonne, Brud Nevez - Emgleo Breiz, Brest. 